\title{
A Novel Approach to Improve the Barrier Properties of PET/Clay Nanocomposites
}

\author{
Kazem Majdzadeh-Ardakani, Shahab Zekriardehani, \\ Maria R. Coleman, and Saleh A. Jabarin \\ Polymer Institute and Department of Chemical and Environmental Engineering, University of Toledo, Toledo, OH 43606-3390, USA \\ Correspondence should be addressed to Saleh A. Jabarin; saleh.jabarin@utoledo.edu
}

Received 19 February 2017; Revised 11 April 2017; Accepted 18 April 2017; Published 11 May 2017

Academic Editor: Hossein Roghani-Mamaqani

Copyright (C) 2017 Kazem Majdzadeh-Ardakani et al. This is an open access article distributed under the Creative Commons Attribution License, which permits unrestricted use, distribution, and reproduction in any medium, provided the original work is properly cited.

\begin{abstract}
An investigation of oleic acid-modified clay versus plain clay with regard to the physical and barrier properties of PET/clay nanocomposites was performed. Montmorillonite (MMT) and Cloisite 30B nanoclays were modified by long-chain oleic acid and identified as ol-MMT and ol-30B, respectively. Fourier Transformed Infrared Spectroscopy and X-ray diffraction (XRD) results revealed that the fatty acid was associated with the clay surface and that the gallery spacing of the layered silicates was expanded. In the case of ol-MMT, a disordered structure of layered silicates was achieved. TGA results indicated that ol-MMT showed thermal stability and could survive PET processing temperature. The degradation of ol-30B, however, increased after modification because of the presence of oleic acid. PET/clay nanocomposites were prepared with modified ol-MMT and modified ol-30B by using a twin screw extruder. XRD indicated that there was a significant improvement on the dispersion of nanoclays modified with long-chain oleic acid into the PET matrix, and an exfoliated structure was achieved. DSC data also revealed that crystallization behaviors of nanocomposites prepared with oleic acid-modified clays are similar to that of extruded PET. Significant improvements in the mechanical and barrier properties of stretched PET/clay nanocomposites were also achieved.
\end{abstract}

\section{Introduction}

Poly(ethylene terephthalate) (PET) is a semicrystalline thermoplastic which is widely used in the manufacture of fibers, films, and beverage containers. Along with its low cost, PET shows chemical and thermal resistance, good optical properties, and good processability [1]. One of the main applications of PET in the packaging industries is its usage in the manufacture of plastic bottles for carbonated soft drinks, water, and juice [2]. There are two common approaches for improving the barrier properties of PET to be used for highly oxygen-sensitive food packaging. These are passive barrier techniques achieved by adding fillers to the polymer matrix to create a tortuous pass for gas molecules $[3,4]$ and active barrier techniques that include oxygen scavengers in the polymer matrix $[5,6]$.

Polymer/clay nanocomposites have attracted significant interest in recent years, because they provide advantages compared to conventional composites. Introducing small amounts of nanoclays into the polymer matrix is one of the passive barrier methods used to further improve thermal stability, barrier, and mechanical properties of PET [7-11]. These improvements, however, are greatly influenced by the degree of clay dispersion into the polymer matrix. Preparing nanocomposites with an exfoliated structure leads to better barrier and mechanical properties [12, 13]. Pristine montmorillonite (MMT) and commercially modified clays have been widely used in areas of nanocomposite research. The compatibility of pristine clay (MMT) with most engineering polymers is very low. It is essential to use clays modified with organic surfactants in order to make them compatible with the polymer matrix. Most of the recent efforts, therefore, have focused on modification of MMT with organic surfactants [7, 14-17]. Commercial nanoclays modified with quaternary ammonium surfactants have widely been used in the preparation of nanocomposites. These 
nanoclays, however, degrade at PET processing temperatures $\left(270-300^{\circ} \mathrm{C}\right)$. Thermally stable surfactants such as imidazolium $[7,14,15,18]$, quinolinium [19], phosphonium [7, 8, 16], and pyridinium surfactants [17] have been used by some researchers. The results indicate that in most cases a combination of intercalated and exfoliated morphologies has been achieved and the uniform dispersion of nanoclays into the PET matrix has remained inaccessible. This is because of the lack of compatibility of modified clays with PET and difficulty in diffusing the long polymer chains into the gallery spacing of layered silicates. Consequently, there has been no significant improvement in the barrier properties of PET nanocomposites.

In an active barrier approach, a component such as an oxygen scavenger is added to PET to react with any oxygen present and protect the packaged food against quality changes. Bio-based unsaturated fatty acids such as oleic acid (with a single double bond) can be used as oxygen scavengers to improve the barrier properties of PET $[20,21]$.

In this work, the main objective has been to develop a new method for preparation of exfoliated PET/clay nanocomposites with superior barrier properties. The approach is to combine both passive barrier and active barrier techniques by modifying pristine clay and commercially modified clay (Cloisite 30B) with long-chain fatty acids and blending these nanoparticles with PET to make nanocomposites with superior barrier properties. This modification not only opens up the gallery spacing of layered silicates and makes them more accessible for PET to diffuse inside the galleries but also provides an oxygen scavenger that can react with oxygen and protect foods and beverages from oxidation. No published reports have been found describing the introduction of fatty acids as oxygen scavengers into nanoclays to improve the dispersion and barrier properties of PET/clay nanocomposites by using both active and passive barrier approaches.

\section{Experimental}

2.1. Materials. PET resin (grade HP806) with an intrinsic viscosity (IV) of $0.84 \mathrm{dL} / \mathrm{g}$ was obtained from Wellman Company (Bay St. Louis, MS). MMT and Cloisite 30B clays, with respective cation exchange capacities of 92.6 and $90 \mathrm{meq} / 100 \mathrm{~g}$, were generously donated by Southern Clay Products (Gonzales, TX). Oleic acid (technical grade, 90\%) was purchased from Sigma-Aldrich (Allentown, PA). Methanol was obtained from Fisher Scientific (Chicago, IL).

2.2. Modification of Nanoclays with Oleic Acid. The organically modified clays (ol-MMT and ol-30B) were synthesized by mixing MMT or Cloisite 30B with oleic acid. To prepare olMMT and ol-30B, mixtures of the clays and fatty acids $(1: 2$ by mass) were prepared and blended in a Parr Reactor with a fixed rotor speed of $50 \mathrm{rpm}$ and temperature of $60^{\circ} \mathrm{C}$. These mixtures were stirred for 2 hours before being removed and washed repeatedly with methanol to remove unbounded fatty acids. Modified samples were vacuum-dried at $90^{\circ} \mathrm{C}$ for 24 hours and then ground into a fine powder.

Water molecules encapsulate sodium in the interlayer of MMT and they can react with the $\mathrm{C}=\mathrm{O}$ groups of the fatty acid. A bridge can also be formed between water molecules and metal ions present in the layer structure. Another possibility is the interaction between free $\mathrm{OH}$ groups at the surface of the clay particles and carboxyl group of fatty acid. The quaternary amines with long alkyl chain present in the interlayer space of Cloisite 30B, expanded the silicate layers, and facilitate the way for intercalation of long-chain fatty acids during mixing. Furthermore, the presence of the long alkyl chains in the interlayer of clay particles reduces the hydrophilic character of these particles and makes it easier for fatty acid alkyl chains to be drawn inside the clay interlayer space due to the strong van der Waals force of attraction. The possibility of interaction between the ammonium head groups of quaternary ammonium surfactants and the carboxylic acid of oleic acid via hydrogen bonding could also be speculated [22].

2.3. Preparation of Nanocomposites. PET and modified clays ( $2 \%$ wt.\%) were melt blended with a corotating twin screw extruder at $280^{\circ} \mathrm{C}$ and $200 \mathrm{rpm}$. Before extrusion, PET samples were dried in a Conair dehumidifying air dryer at $140^{\circ} \mathrm{C}$ overnight and the modified clays were vacuum-dried at $100^{\circ} \mathrm{C}$ overnight. Before loading the mixture of PET and clay into the hopper of the extruder, PET and the dry modified clays were mixed in a sealed container for $3 \mathrm{~min}$. Nanocomposite strands extruded from the die were quenched through a water bath and then cut into pellets with a pelletizer and vacuumdried for further characterization.

\subsection{Preparation of Nanocomposite Films. An ALLROUND-} ER 320 s Arburg Injection Molding Machine (55-ton capacity) was used to prepare PET nanocomposite preforms. Before the injection molding process, the samples were dried in a Conair dehumidifying air dryer at $140^{\circ} \mathrm{C}$ overnight.

The 2 wt.\% PET nanocomposite preforms obtained by the injection molding process were stretched and blown into bottles using a lab-scale stretch-blow molding machine. In this process, infrared heaters provide an appropriate preform temperature profile. An axial stretch rod was used for stretching the preforms to a desirable length. Then, the final pressure or the full blow pressure was applied, which inflated the bottle to the desired volume (diameter and length of the bottles were $10.5 \mathrm{~cm}$ and $30 \mathrm{~cm}$, resp.). This orientation process leads to the strain-induced crystallization and strain hardening of the stretched polymer $[23,24]$. The bottles were solidified and cooled when they attained the shape of the mold. The sidewalls of the stretch blow molded bottles were used to investigate the tensile and oxygen barrier properties for nanocomposites and base PET.

\section{Characterization}

3.1. Attenuated Total Reflection-Fourier Transform Infrared Spectroscopy (ATR-FTIR). Fourier Transformed Infrared (FTIR) spectra of modified clays were taken with a PerkinElmer 1600 spectrometer in the range of $700-4000 \mathrm{~cm}^{-1}$ to confirm that oleic acid was bonded to the clay surface after modification. Attenuated total reflection (ATR) is a sampling technique used in conjunction with infrared spectroscopy to 


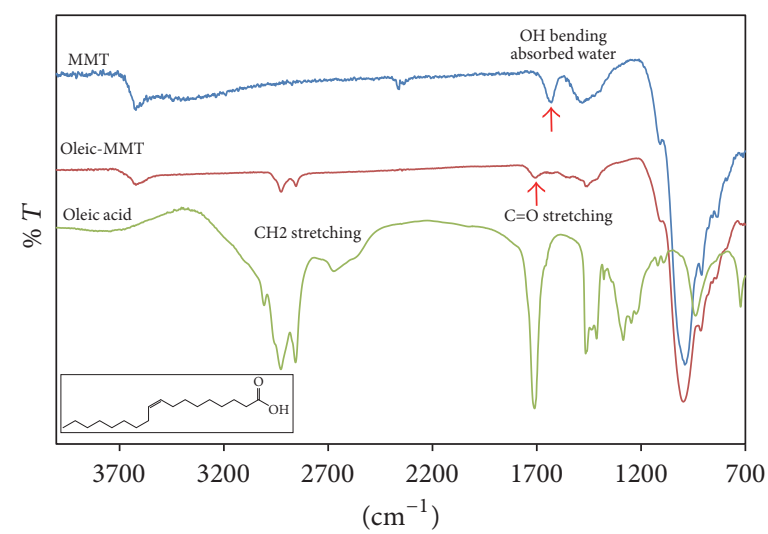

FIGURE 1: FTIR spectra of oleic acid, MMT, and modified clay (ol-MMT).

examine the samples directly in the solid or liquid state without further preparation.

3.2. Wide Angle X-Ray Diffraction (WAXD). A Rigaku Ultima $\mathrm{X}$-ray diffractometer (The Woodlands, TX) with a Ni-filtered $\mathrm{Cu}$ Ka radiation ( $0.1541 \mathrm{~nm}$ wavelength) generated at $44 \mathrm{kV}$ and $44 \mathrm{~mA}$ was used to determine the basal spacing of clays and their nanocomposites using the Bragg equation $(\lambda=$ $2 d \sin \theta$ ), where $\theta$ is the diffraction angle, $\lambda$ is the wavelength, and $d$ is the basal spacing of clays. Samples were scanned at $0.5^{\circ} / \mathrm{min}$ in the range of $2 \theta=1-8^{\circ}$.

3.3. Thermal Gravimetric Analysis (TGA). Thermal Gravimetric Analysis (TGA) was conducted on a Q500 TGA instrument under nitrogen atmosphere. The samples of 5-10 $\mathrm{mg}$ were heated from room temperature to $700^{\circ} \mathrm{C}$ at a rate of $10^{\circ} \mathrm{C} / \mathrm{min}$.

3.4. Rheology and Melt Viscosity. Melt viscosity and rheological measurements of nanocomposites were determined using a parallel-plate Rheometric Scientific (RDA III) dynamic analyzer (Piscataway, NJ). Vacuum-dried samples were melted between parallel disks (diameter: $2.5 \mathrm{~cm}$ ) at $270^{\circ} \mathrm{C}$ in the presence of nitrogen at $15 \%$ strain amplitude. Intrinsic viscosities (IV) of PET samples with different molecular weights were measured by dilute solution viscometry method [25] and the corresponding melt viscosity was measured by rheometer. Afterwards, a calibration curve was created by plotting IV versus $\ln \eta^{*}$ in order to determine the intrinsic viscosity from the corresponding melt viscosity.

3.5. Differential Scanning Calorimeter (DSC). A PerkinElmer DSC 7 (Shelton, CT) was applied to measure the thermal characteristics of extruded PET and its nanocomposites in a nitrogen atmosphere. Dried samples (at $140^{\circ} \mathrm{C}$ for $12 \mathrm{hrs}$ ) of about $10 \mathrm{mg}$ were heated at a rate of $10^{\circ} \mathrm{C} / \mathrm{min}$ from 40 to $300^{\circ} \mathrm{C}$

3.6. Tensile Testing. An Instron 4400R tester was used to determine elastic modulus, stress, and strain at break of "dog-bone" shaped specimens (from the sidewalls of the
PET bottles) according to ASTM D638 type IV. All the test samples were held for one week at $50 \%$ humidity and $23^{\circ} \mathrm{C}$ temperature before evaluation. Samples were stretched at a crosshead speed of $2 \mathrm{in} / \mathrm{min}$ during the test. Five specimens were measured for each evaluated sample type and averaged.

3.7. Oxygen Permeability. The oxygen permeability test was carried out on the sidewalls of the PET and nanocomposite bottles. This test was done by a MOCON OX-TRAN 1050 tester according to ASTM D3985. The experiment conditions were $23^{\circ} \mathrm{C}$ and $45 \%$ relative humidity. The exposed area and the thickness of the films were $50 \mathrm{~cm}^{2}$ and $10-13$ mil, respectively. A mixture of $98 \% \mathrm{~N}_{2}$ and $2 \% \mathrm{H}_{2}$ was used as a carrier gas. The instrument was calibrated with a standard Mylar polyester film before sample measurements.

\section{Results and Discussion}

4.1. ATR-FTIR Results. FTIR spectra of oleic acid, MMT, and MMT modified with oleic acid (ol-MMT) are shown in Figure 1. In the spectrum of MMT, the peak at $3619 \mathrm{~cm}^{-1}$ is related to $\mathrm{O}-\mathrm{H}$ stretching. The $\mathrm{Si}-\mathrm{O}$ out-of-plane and inplane stretching bonds are observed at $1110 \mathrm{~cm}^{-1}$ and 996 $\mathrm{cm}^{-1}$, respectively. The $\mathrm{Al}-\mathrm{O}$ and $\mathrm{Mg}-\mathrm{O}$ stretching bonds are assigned to the peaks between 836 and $910 \mathrm{~cm}^{-1}$. The strong peak at $1630 \mathrm{~cm}^{-1}$ is assigned to the bending mode of absorbed water $[26,27]$.

The spectrum of oleic acid shows a peak at $1710 \mathrm{~cm}^{-1}$ which is related to the vibrations of $\mathrm{C}=\mathrm{O}$ groups. Two peaks at $2932 \mathrm{~cm}^{-1}$ and $2856 \mathrm{~cm}^{-1}$ are related to the symmetric and asymmetric vibrations of methylene $\left(-\mathrm{CH}_{2}\right)$ groups present in the fatty acid [26]. Characteristic peaks of the oleic acid are included in the spectra of the modified clay, indicating the presence of oleic acid in the gallery spacing of the nanoclays. These peaks are indicative of surfactant associated with the clay surface because the unbounded fatty acids were removed by washing with methanol. Since the vibrations of $\mathrm{C}=\mathrm{O}$ groups remain the same $\left(1710 \mathrm{~cm}^{-1}\right)$ for oleic acid and ol-MMT samples, the possibility of the interaction between water molecules and metal ion present in the layer structure via a water bridge is speculated. The 
TABLE 1: IR bands of oleic acid, MMT, 30B, ol-MMT, and ol-30B.

\begin{tabular}{|c|c|c|c|c|c|}
\hline \multicolumn{5}{|c|}{ Wavenumber $\left(\mathrm{cm}^{-1}\right)$} & \multirow{2}{*}{ Vibration } \\
\hline Oleic acid & MMT & Ol-MMT & $30 \mathrm{~B}$ & Ol-30B & \\
\hline $3500-3800$ & 3619 & 3619 & 3619 & 3619 & $\mathrm{O}-\mathrm{H}$ stretching \\
\hline 3016 & - & - & - & 3016 & $\mathrm{C}-\mathrm{H}$ stretching in $\mathrm{C}=\mathrm{C}-\mathrm{H}$ \\
\hline 2856,2932 & - & 2856,2932 & 2856,2932 & 2856,2932 & Aliphatic C-H \\
\hline 1710 & - & 1710 & - & 1738 & $\mathrm{C}=\mathrm{O}$ stretching \\
\hline - & 1630 & - & 1630 & - & $\mathrm{O}-\mathrm{H}$ bending \\
\hline 1460 & 1460 & 1460 & 1460 & 1460 & In-plane $\mathrm{O}-\mathrm{H}$ band \\
\hline 1412 & - & 1412 & - & 1412 & Methyl $\left(-\mathrm{CH}_{3}\right)$ group \\
\hline - & - & - & 1457 & 1457 & $\mathrm{CH}_{2}$ bending \\
\hline 1284 & - & - & - & 1312 & $\mathrm{C}-\mathrm{O}$ stretching \\
\hline- & 1110,996 & 1110,996 & 1110,996 & 1110,996 & $\mathrm{Si}-\mathrm{O}$ out-of-plane and in-plane stretching \\
\hline 937 & - & - & - & - & Out-of-plane $\mathrm{O}-\mathrm{H}$ stretching \\
\hline - & 910,836 & 910,836 & 910,836 & 910,836 & $\mathrm{Al}-\mathrm{O}$ and $\mathrm{Mg}-\mathrm{O}$ stretching \\
\hline
\end{tabular}

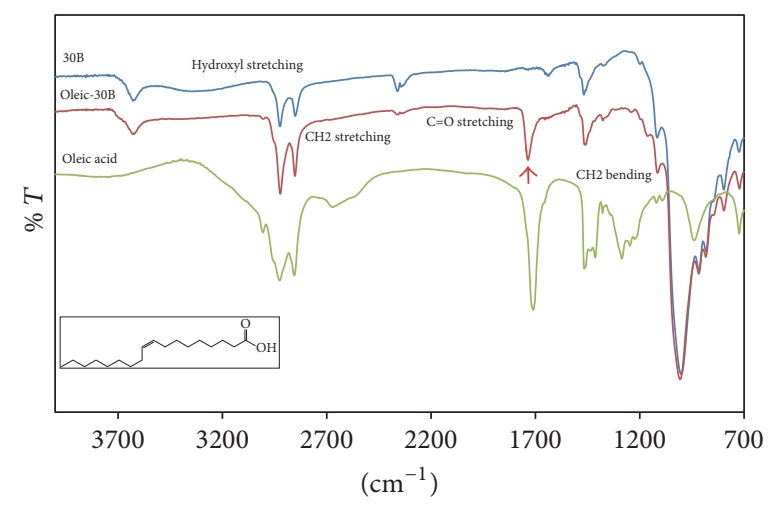

FIGURE 2: FTIR spectra of oleic acid, 30B, and modified clay (ol-30B).

absence of $\mathrm{O}-\mathrm{H}$ bending peak in the modified clay spectrum suggests that, with surfactant loading, MMT was transformed into an entirely hydrophobic material.

FTIR spectra of oleic acid, Cloisite 30B, and Cloisite 30B modified with oleic acid (ol-30B) are shown in Figure 2. Most of the peaks are the same in these components because of the organic surfactant present in the interlayer of 30B. In the spectrum of oleic acid, the peak at $3016 \mathrm{~cm}^{-1}$ is related to the double bond $(\mathrm{C}=\mathrm{C})$. This peak appeared in the spectrum of ol-30B. It can also be observed that the peak related to the $\mathrm{C}=\mathrm{O}$ of the oleic acid has shifted to the higher wavenumbers $\left(1738 \mathrm{~cm}^{-1}\right)$, indicating the strong interaction between the $\mathrm{C}=\mathrm{O}$ group and quaternary ammonium surfactant present in the interlayer of Cloisite 30B [28]. This shift could be an indicative of changing the carboxylic acid group to an ester group. The possibility of interaction between either the ammonium head groups or $\mathrm{OH}$ groups of quaternary ammonium surfactants and the carboxylic acid group of oleic acid via hydrogen bonding could be speculated.

Table 1 summarizes the IR bands of oleic acid, MMT, 30B, and the modified clays.
4.2. XRD Results. XRD results obtained for pristine MMT and ol-MMT are shown in Figure 3. The unmodified clay shows a characteristic peak at $2 \theta=7.0^{\circ}$, indicating $d$-spacing of $1.27 \mathrm{~nm}$. The characteristic peak disappeared after modification of clay with the fatty acid. This indicates that oleic acid molecules could diffuse into the gallery spacing of pristine clay and interact with $\mathrm{Na}^{+}$contained inside its interlayer via hydrogen bonding. This modification, therefore, could change the arrangement of layered silicates and make them disordered. This is an important achievement since the polymer chains should be able to more easily diffuse into the gallery spacing of the nanoclay during preparation of PET nanocomposites. Few studies have been reported with regard to achieving a disordered arrangement of nanoclays after the modification process.

XRD results of Cloisite $30 \mathrm{~B}$ and ol-30B are shown in Figure 4. Commercial 30B (clay modified with methyl tallow bis-2-hydroxyethyl ammonium cations) has $d$-spacing of $1.88 \mathrm{~nm}$. Modification of clay with oleic acid resulted in a shift in the (001) peak by $2.6^{\circ}$. This corresponds to an increase in $d$-spacing of about $2.36 \mathrm{~nm}$, indicating that the oleic acid molecules are intercalated into the clay galleries. This is 


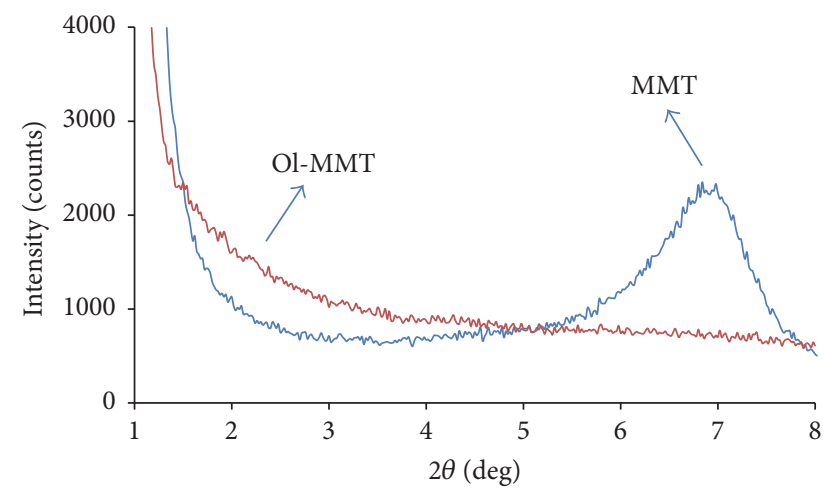

FIgURE 3: XRD results of MMT and ol-MMT.

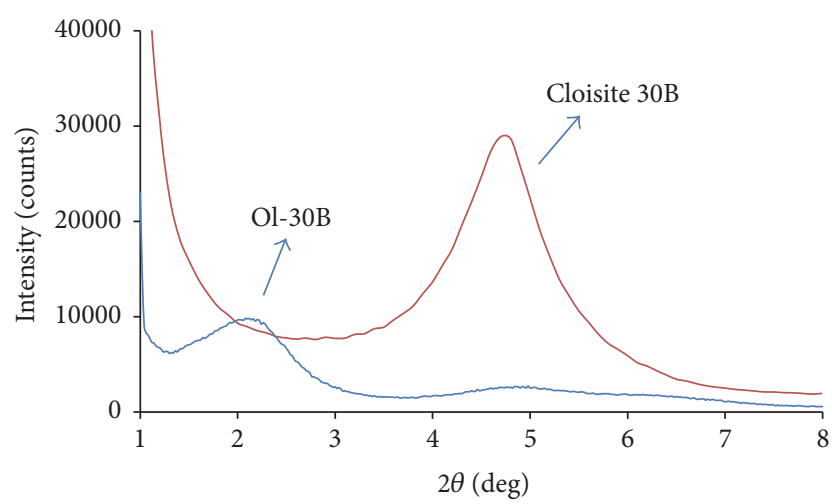

FIgURE 4: XRD results of $30 \mathrm{~B}$ and ol-30B.

also a large increase of the gallery spacing of nanoclays. Because of this large gallery spacing and compatibility of ol$30 \mathrm{~B}$ with PET, good dispersion of these nanoparticles into the PET matrix is expected during the process of preparing nanocomposites.

4.3. TGA Results. The percentage mass loss with temperature plots for oleic acid, MMT, and, ol-MMT is shown in Figure 5. There was no significant mass loss for unmodified clay up to $570^{\circ} \mathrm{C}$. Evaporation of free water in clay, which is hydrophilic, results in an initial mass loss below $200^{\circ} \mathrm{C}$. Evaporation of structural water under dehydroxylation causes a mass loss between 570 and $700^{\circ} \mathrm{C}$ in the unmodified clay $[16,29]$.

Since the boiling point of oleic acid is about $250^{\circ} \mathrm{C}$, the percentage mass loss increased from 0 to $100 \%$ very quickly. MMT modified with oleic acid showed no significant mass loss up to $250^{\circ} \mathrm{C}$. This may indicate that the fatty acid had penetrated inside the gallery spacing of layered silicates and made them more hydrophobic (the initial degradation, below $100^{\circ} \mathrm{C}$, for ol-MMT is much less than that of MMT). The onset of decomposition temperature $\left(T_{0}\right)$ value of ol-MMT is $250^{\circ} \mathrm{C}$, which corresponds with the boiling point of oleic acid. Therefore, the thermal stability of modified MMT decreased in the presence of oleic acid. The degradation of modified clay is, however, a slow process because of the strong bonds between clay particles and fatty acid molecules. From the results, it can be concluded that the modification process was

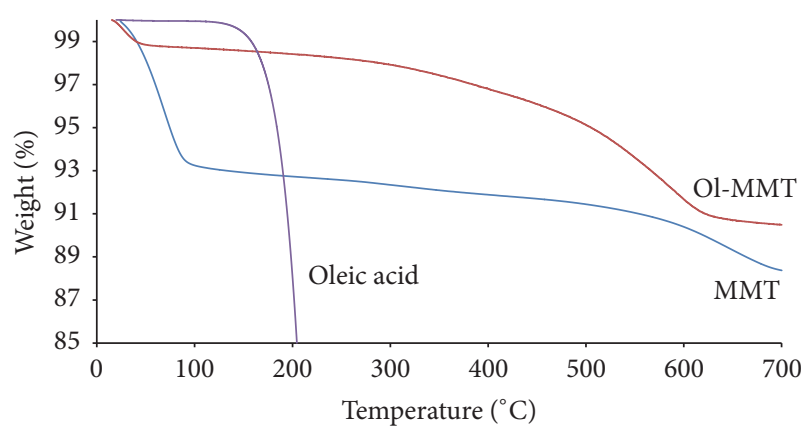

FIGURE 5: TGA results of oleic acid, MMT, and ol-MMT.

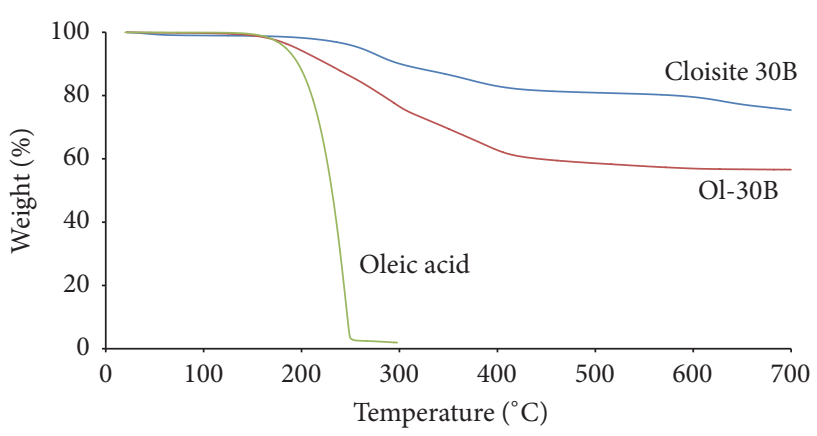

FIgURE 6: TGA results of oleic acid, 30B, and ol-30B.

successful and clays modified with oleic acid could survive PET processing temperatures of $\sim 300^{\circ} \mathrm{C}$.

In the case of Cloisite 30B organoclays shown in Figure 6, the major mass loss occurred in the temperature range of $180-420^{\circ} \mathrm{C}$. Quaternary ammonium ions present in $30 \mathrm{~B}$ clays started to degrade at the onset of decomposition temperature $\left(T_{0}=180^{\circ} \mathrm{C}\right)$.

Modification of Cloisite 30B with oleic acid reduced $T_{0}$ value of ol-30B to $160^{\circ} \mathrm{C}$. Faster and more degradation of modifiers, therefore, occurred in the case of ol-30B. This indicated that fatty acid was intercalated inside the gallery spacing of the layered silicates. Although the decomposition temperature of the modifiers is below the PET processing temperature, the very large gallery spacing of $30 \mathrm{~B}$ modified with fatty acid along with high affinity between organic modifiers and PET should facilitate the diffusion of polymer chains into the interlayers of modified clays.

4.4. Morphology of Nanocomposites. XRD data for $2 \mathrm{wt} . \%$ PET/MMT, PET/ol-MMT, and PET/ol-30B nanocomposites are shown in Figure 7. PET/ol-MMT is nanocomposite prepared with pristine clay modified with oleic acid, while PET/ol-30B is nanocomposite prepared with Cloisite 30B modified with oleic acid. A characteristic diffraction peak appeared at $2 \theta=6.1^{\circ}$ with $d$-spacing of about $1.4 \mathrm{~nm}$ for $2 \mathrm{wt} . \%$ PET/MMT nanocomposites, indicating the agglomeration of nanoparticles in the PET. The presence of flat curves for both PET/ol-MMT and PET/ol-30B samples indicates that exfoliated structures may have been obtained. Modification of nanoclays by fatty acid, therefore, appears to have resulted in an exfoliated structure for these PET/clay nanocomposites. In 


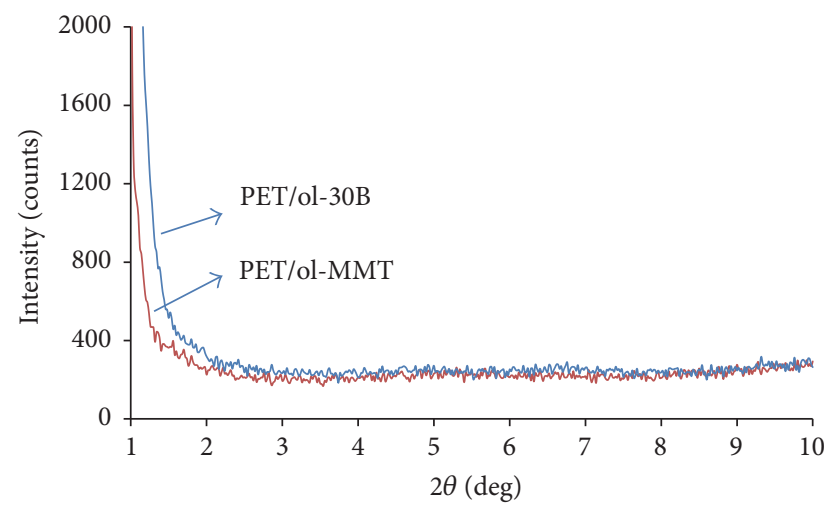

FIGURE 7: XRD results of $2 \mathrm{wt} . \%$ PET/ol-MMT and PET/ol-30B nanocomposites.

fact, the quaternary amines and fatty acids with long alkyl chain present in the interlayer space of 30B facilitated the intercalation of PET chains during the melt compounding process. Moreover, according to the FTIR results, the presence of the long alkyl chains in the interlayer space reduced the hydrophilic character of the clay and because of the interaction between PET and fatty acid molecules, guest polymer chains were drawn inside the clay interlayer space.

TEM was used to verify the results obtained from XRD data. Micrographs of 2 wt.\% PET/ol-MMT and PET/ol-30B nanocomposites are shown in Figures 8 and 9. Since the original micrographs (left side) are vague, a program was used to make a better contrast between clay particles and polymer matrix. The processed micrographs are shown in the right side. It can be seen that the particles were well dispersed into the PET matrix. There are, however, some agglomerations of clay particles, especially in the case of $2 \%$ PET/ol-MMT sample. These TEM results indicate that modification of clays with fatty acids provides a combination of exfoliated/ intercalated morphologies for PET/clay nanocomposites.

4.5. Rheology and Melt Viscosity. Melt viscosity results and corresponding PET equivalent IV values for extruded PET and 2 wt.\% nanocomposites are shown in Figure 10 and Table 2. The complex viscosity decreased with increasing angular frequency for all the samples. The maximum value of complex viscosity was observed for extruded PET sample. The 2 wt.\% PET/MMT nanocomposite exhibited lower complex viscosity and IV values compared to the $2 \mathrm{wt} . \%$ PET/olMMT nanocomposite. The modification of nanoclays with oleic acid improved the dispersion of nanoparticles into the PET matrix. These well-dispersed nanoparticles can present a shielding effect which reduces the mass transport rates during the degradation process in the polymer matrix [30]. The complex viscosity of PET/ol-MMT is, therefore, very close to that of extruded PET. PET/ol-30B nanocomposites exhibited lower complex viscosity and IV values compared to the PET/ ol-MMT. As shown in Figure 6, presence of fatty acids in Cloisite 30B causes thermal instability in ol-30B. As a result, degradation of the polymer matrix was accelerated by the degradation of organic modifier present in ol-30B nanoparticles. The complex viscosity and IV values of PET/ol-30B were
TABLE 2: IV values of PET and nanocomposites.

\begin{tabular}{lc}
\hline Sample & IV (dl/g) \\
\hline Pure PET & $0.81 \pm 0.01$ \\
Extruded PET & $0.64 \pm 0.01$ \\
2.0 wt.\% PET/MMT & $0.50 \pm 0.01$ \\
2.0 wt.\% PET/ol-MMT & $0.62 \pm 0.02$ \\
2.0 wt.\% PET/30B & $0.51 \pm 0.03$ \\
2.0 wt.\% PET/ol-30B & $0.57 \pm 0.02$ \\
\hline
\end{tabular}

higher than those of the PET/30B samples. This confirms the improvement in the dispersion of 30B nanoparticles and their shielding effect after modification with oleic acid. PET/MMT and PET/30B, however, exhibited the same behavior and IV values. In fact, the degradation of polymer matrix was accelerated by the agglomerations of unmodified MMT and 30B nanoparticles. These agglomerations increased the mass transport rate during the degradation process in the polymer. This, therefore, causes a reduction in the complex viscosity values of composites. It can be concluded that although $30 \mathrm{~B}$ shows better compatibility with PET, its thermal degradation causes it to behave like an unmodified clay.

The storage modulus $\left(G^{\prime}\right)$ and loss modulus $\left(G^{\prime \prime}\right)$ versus frequency for all samples are shown in Figures 11 and 12. Samples prepared with unmodified nanoclays showed lower storage modulus because of the lower level of clay dispersion. On the other hand, extruded PET, PET/ol-MMT, and PET/ol$30 \mathrm{~B}$ nanocomposites exhibited higher storage moduli as a result of more interconnected structure and geometric constraints. The storage modulus of PET/ol-MMT is very close to that of extruded PET. Poorer dispersion of MMT and $30 \mathrm{~B}$ particles accelerated the degradation process in the PET matrix; hence the storage modulus of PET/MMT and $\mathrm{PET} / 30 \mathrm{~B}$ nanocomposites is lower than that of PET/ol-MMT and PET/ol-30B. Although the storage modulus of PET/30B is higher than that of PET/MMT at lower frequencies because of better affinity with PET, their $G^{\prime}$ values would be similar at higher frequencies, where the behavior of the matrix is dominant.

4.6. Thermal Analysis of Nanocomposites. Thermal characteristics of extruded PET and its nanocomposites are presented in Table 3. Results show that the glass transition temperature $\left(T_{g}\right)$ and melting temperature $\left(T_{m}\right)$ of all nanocomposites samples are very close to those of extruded PET. The enthalpy of melting $\left(\Delta H_{m}\right)$ values of nanocomposites is slightly higher than that of extruded PET. According to Table 3 , in the case of PET nanocomposites, crystallization occurred during the quenching step and $\Delta H_{c}{ }^{m}$ was low compared to that of PET.

As shown by the cooling process data given in Table 3 , the crystallization peak temperature $\left(T_{c}^{c}\right)$ and the onset of crystallization temperature $\left(T_{\mathrm{on}}{ }^{c}\right)$ of nanocomposites are slightly higher than those of PET, indicating that crystallization in nanocomposites occurs a little bit faster. Half time of crystallization $\left(t_{1 / 2}\right)$, which is also an indicator of the overall crystallization rate corresponding to the effects of both 

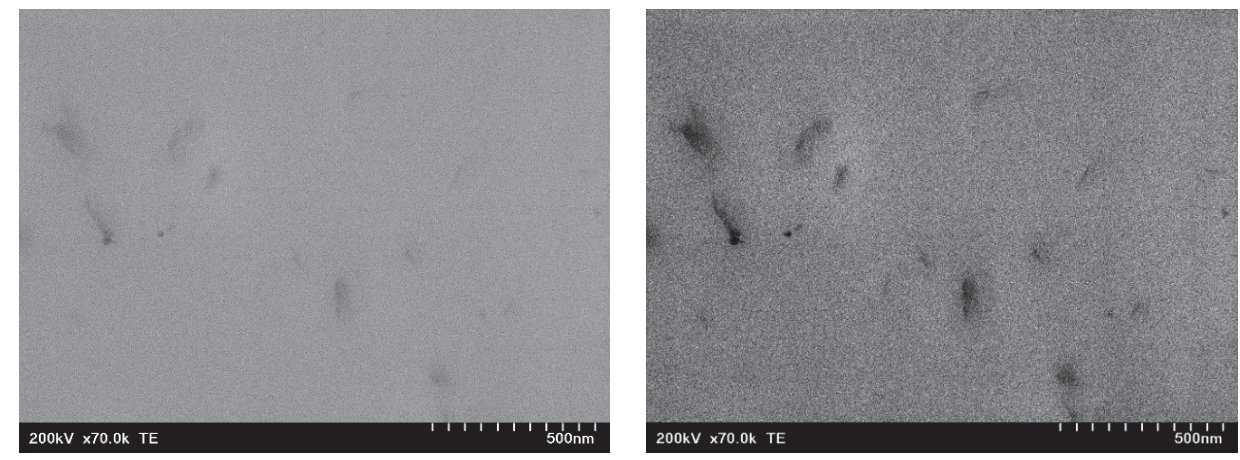

FIGURE 8: TEM micrographs of 2 wt.\% PET/ol-MMT nanocomposite.
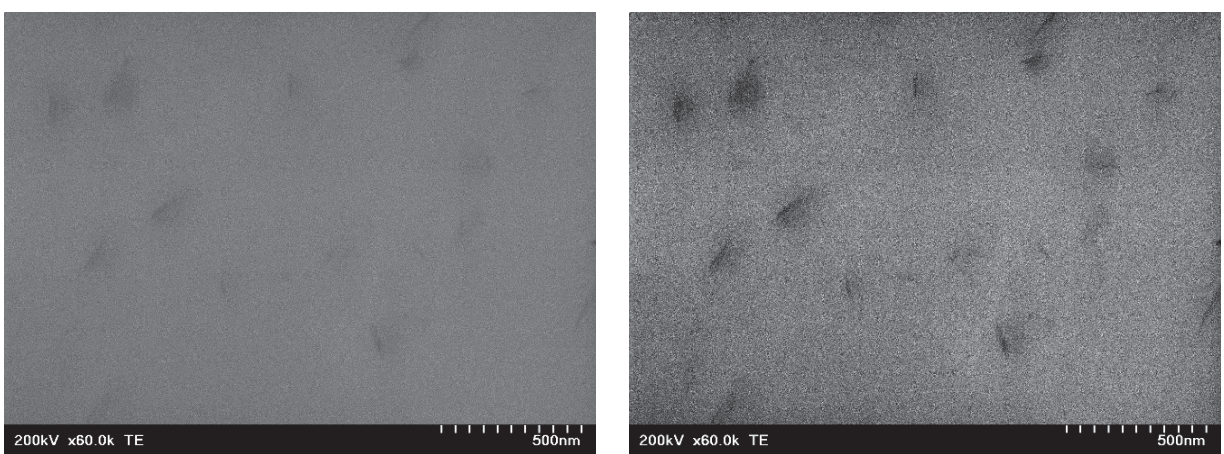

FIgURE 9: TEM micrographs of 2 wt.\% PET/ol-30B nanocomposite.

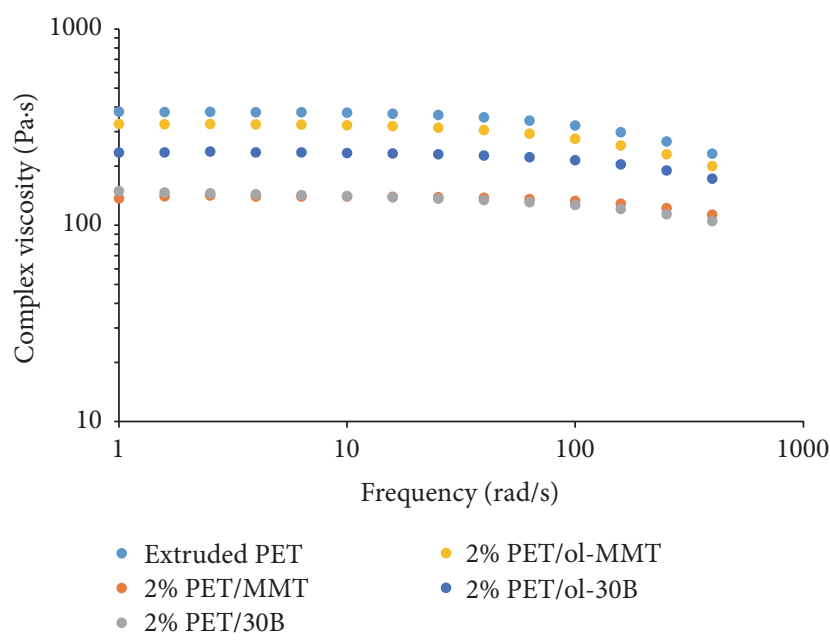

Figure 10: Melt viscosity of PET and nanocomposites.

nucleation and crystal growth, can be measured by using the following equation [31]:

$$
t_{1 / 2}=\frac{\left(T_{\mathrm{on}}{ }^{c}-T_{c}^{c}\right)}{\chi}
$$

where $T_{\text {on }}{ }^{c}$ is the onset of crystallization temperature, $T_{c}^{c}$ is the crystallization temperature at the exothermic peak, and $\chi$ is the cooling rate $\left({ }^{\circ} \mathrm{C} / \mathrm{min}\right)$. According to Table $3, t_{1 / 2}$ values of the nanocomposites are lower than those of extruded PET.

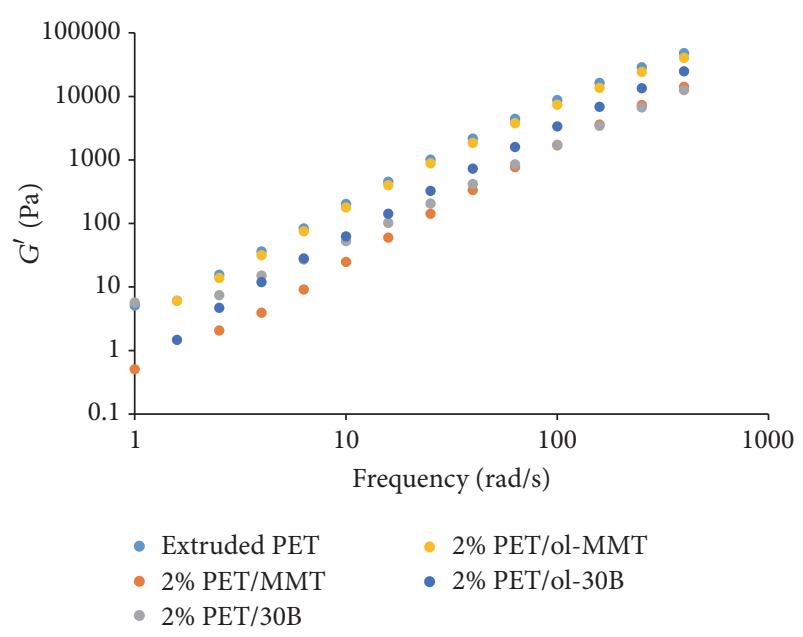

FIGURE 11: Storage modulus of PET and nanocomposites.

$t_{1 / 2}$ values of PET/ol-MMT and PET/ol-30B nanocomposites, however, are higher than those of PET/MMT and PET/30B and closer to those of extruded PET. From these results, it can be concluded that unmodified clay has a significant influence on the crystallization of PET because of the low level of dispersion of these particles into the PET. Cloisite 30B also showed higher rate of crystallization because of the thermal degradation of organic modifier. Modification of MMT and Cloisite 30B with oleic acid, however, has a smaller effect on the crystallization of polymer because of the improvement in 
TABLE 3: DSC results of 2 wt.\% PET/MMT, PET/ol-MMT, PET/30B, and PET/ol-30B nanocomposites.

\begin{tabular}{|c|c|c|c|c|c|c|c|c|}
\hline \multirow{2}{*}{ Sample } & \multicolumn{4}{|c|}{ Reheating at $10^{\circ} \mathrm{C} / \mathrm{min}$} & \multicolumn{4}{|c|}{ Cooling at $10^{\circ} \mathrm{C} / \mathrm{min}$} \\
\hline & $T_{g}\left({ }^{\circ} \mathrm{C}\right)$ & $\Delta H_{c}^{m}(\mathrm{~J} / \mathrm{g})$ & $T_{m}\left({ }^{\circ} \mathrm{C}\right)$ & $\Delta H_{m}(\mathrm{~J} / \mathrm{g})$ & $T_{c}^{c}\left({ }^{\circ} \mathrm{C}\right)$ & $T_{\text {on }}{ }^{c}\left({ }^{\circ} \mathrm{C}\right)$ & $t_{1 / 2}(\min )$ & $\Delta H_{c}^{c}(\mathrm{~J} / \mathrm{g})$ \\
\hline Extruded PET & 79.1 & 20.7 & 244.1 & 32.8 & 186.2 & 195.3 & 0.91 & 34.4 \\
\hline 2 wt.\% PET/MMT & 79.8 & 5.9 & 245.4 & 37.0 & 192.7 & 199.9 & 0.72 & 38.5 \\
\hline 2 wt. $\%$ PET/ol-MMT & 79.1 & 10.5 & 244.1 & 33.6 & 193.1 & 200.8 & 0.77 & 35.1 \\
\hline 2 wt. $\%$ PET/30B & 78.2 & 13.3 & 244.8 & 35.2 & 193.7 & 201.1 & 0.74 & 36.7 \\
\hline 2 wt.\% PET/ol-30B & 77.6 & 11.2 & 245.1 & 32.8 & 188.8 & 196.6 & 0.78 & 34.0 \\
\hline
\end{tabular}

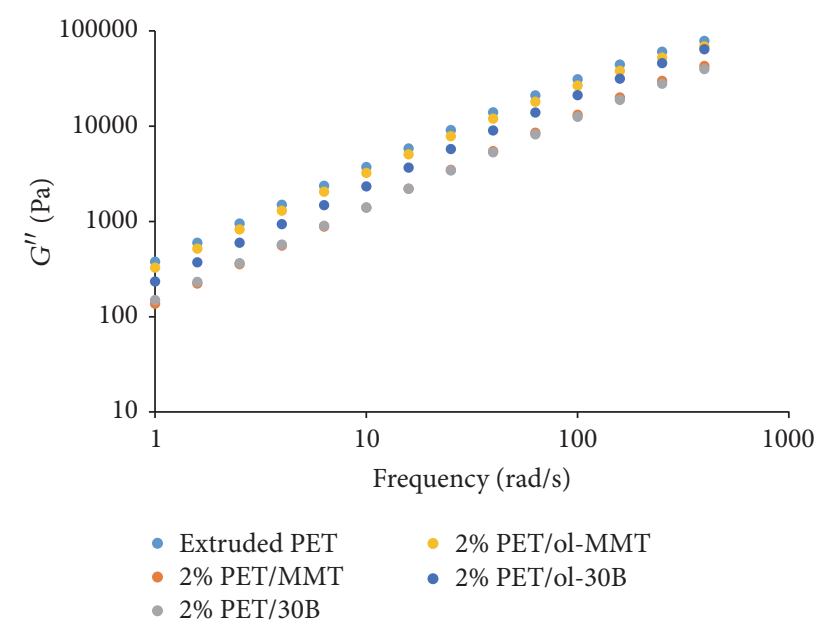

Figure 12: Loss modulus of PET and nanocomposites.

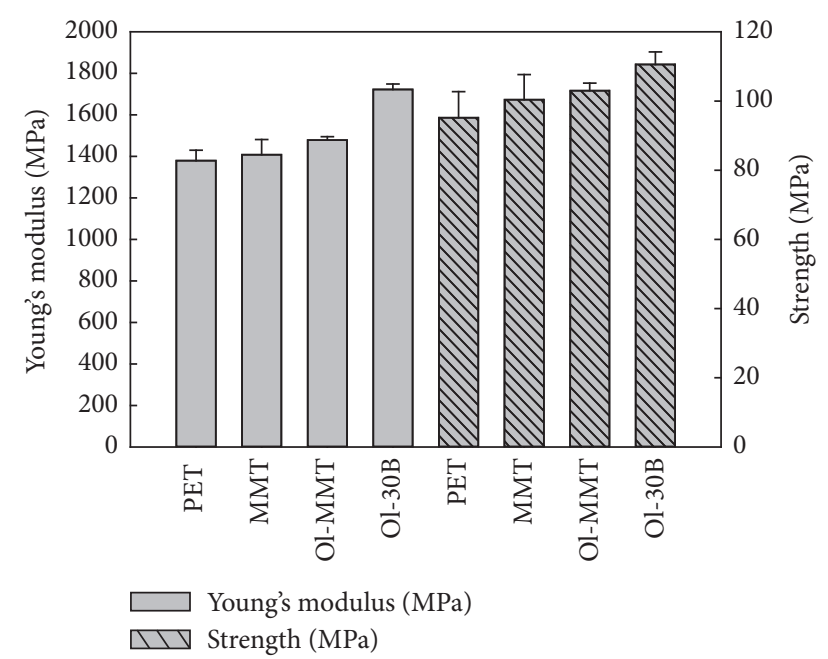

FIGURE 13: Strength of the sidewall of stretched blown PET and nanocomposite bottles.

the dispersion of nanoparticles into the polymer matrix and less amount of agglomeration.

4.7. Mechanical Properties. The strength results obtained for sidewalls of PET and nanocomposite bottles are shown in Figure 13. It can be seen that the PET nanocomposites prepared with both modified clays exhibited higher strength values

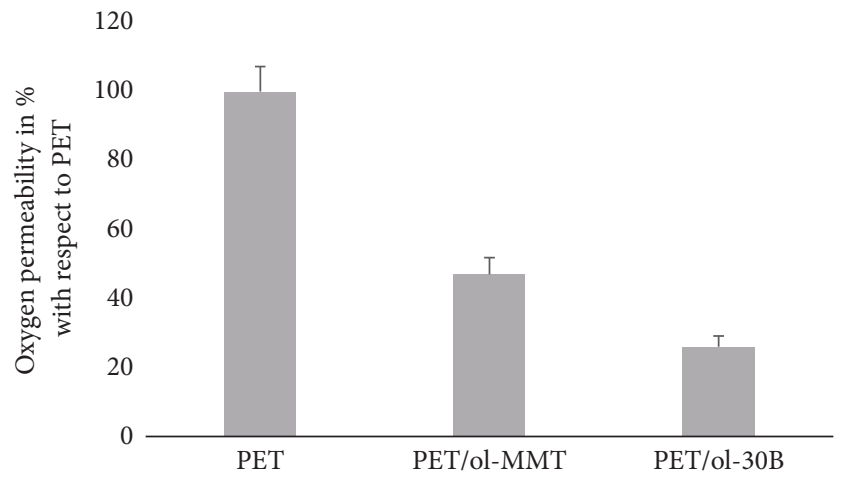

FIGURE 14: Barrier properties of the sidewalls of stretched blown PET and nanocomposite bottles.

than those of the PET because of the reinforcing effect of the stiff clay particles (8\% and $16 \%$ improvement for $2 \mathrm{wt} . \% \mathrm{PET} /$ ol-MMT and 2 wt.\% PET/ol-30B, resp.). The nanocomposites prepared with unmodified clay ( 2 wt.\% PET/MMT), however, showed mechanical property improvement of only $5 \%$.

Young's modulus results for sidewalls of PET and nanocomposite bottles are also presented in Figure 13. PET nanocomposites prepared with both modified clays exhibited higher Young's modulus values than the PET (7\% and 25\% improvement for $2 \mathrm{wt} \%$ PET/ol-MMT and $2 \mathrm{wt} \%$ PET/ol$30 \mathrm{~B}$, resp.). The nanocomposites prepared with unmodified clay ( 2 wt.\% PET/MMT), however, showed only $2 \%$ improvement in their Young's modulus values.

4.8. Barrier Properties. Oxygen permeability results recorded for the sidewalls of PET and nanocomposite bottles are shown in Figure 14. It can be seen that there are $53 \%$ and $74 \%$ improvements in oxygen permeability for $2 \mathrm{wt} . \% \mathrm{PET} / \mathrm{ol}-$ MMT and 2 wt.\% PET/ol-30B nanocomposites, respectively. In fact, the well-dispersed modified clays created a tortuous path for gas molecules and reduced the permeability of the samples. Although phase separation upon stretching of the samples is one of the challenges that researchers faced [32], the good adhesion between the clay particles and PET matrix, in this study, resulted in a significant improvement in the barrier properties of stretched blown bottles, even upon stretching of the samples.

Beside the passive barrier effect of modified nanoparticles, fatty acid, intercalated into the interlayer of layered silicates, might act as an oxygen scavenger and reduce the 
TABLE 4: Slope and intercepts of cumulative plots.

\begin{tabular}{lcc}
\hline Sample & Slope $\left(\mathrm{cc} / \mathrm{m}^{2} \cdot \mathrm{mil} \cdot\right.$ day $)$ & Intercept (days) \\
\hline PET & 8.6 & 13 \\
2.0 wt.\% PET/MMT & 3.1 & 13 \\
2.0 wt.\% PET/30B & 2.4 & 13 \\
2.0 wt.\% PET/ol-MMT & 2.9 & 14 \\
2.0 wt.\% PET/ol-30B & 2.0 & 14 \\
\hline
\end{tabular}

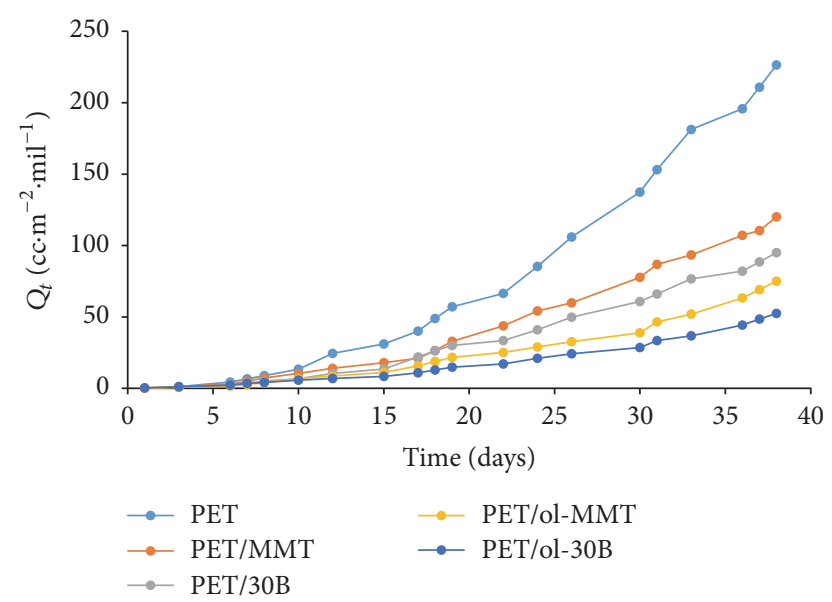

FIGURE 15: Cumulative permeability data for sidewall of stretched blown PET and nanocomposite bottles.

amount of oxygen which passes through the films. In fact, oleic acid includes one double bond which can react with oxygen molecules to reduce the permeability of the samples.

In order to investigate the role of passive and active barrier mechanisms on the permeability of the samples, a measure of cumulative amount of oxygen passing through the films with time was used. The idea of adding active oxygen scavenger into the PET matrix is that the scavenger could delay the induction time period of oxygen transmitted into the PET container and then after some time achieve a steady state similar to that of pure PET. Cumulative plots for PET and PET nanocomposites are shown in Figure 15. The data were collected for 38 days. The slopes of $Q_{t}$ versus time are still changing and they did not achieve a steady state. Therefore, the exact values of induction time are not known yet. However, calculating the slope of $Q_{t}$ versus time between 20 and 38 days gives an indication of the transmission rates. The extrapolated slopes to zero $Q_{t}$ give the values of apparent induction time as shown in Table 4.

In relationship to the unmodified PET, significant reductions in the slopes of the other cumulative plots occurred. These reductions for samples prepared with modified clays are because of the improved compatibility between the clay particles and PET matrix and the good dispersion of these particles into the PET. This also confirmed that the welldispersed modified clays acted as passive barriers and reduced the permeability of the samples. There was, however, no significant change in the intercepts or apparent induction periods of the samples. While it was expected that PET/fatty acid-modified clay nanocomposite may act as an active barrier as well, the data were not carried out to a longer time to confirm the presence of active barrier mechanism. More research is ongoing to confirm the barrier mechanisms in these systems.

\section{Conclusions}

Pristine clay and Cloisite 30B were modified with a longchain fatty acid. FTIR and XRD results revealed that oleic acid could interact with the surface of pristine clay (MMT) and commercially modified clays (Cloisite 30B) to open up their gallery spacing and make them more compatible with PET. This modification increased the gallery spacing of the layered silicates significantly. In the case of MMT modified with oleic acid (ol-MMT), a disordered structure was achieved. TGA results indicated that ol-MMT is thermally stable and can survive PET processing temperatures. There is, however, more degradation in the case of Cloisite 30B modified with oleic acid (ol-30B). XRD indicated that there is a significant improvement on the dispersion of nanoclays modified with long-chain oleic acid into the PET matrix, and an exfoliated structure was achieved. DSC data also revealed that crystallization behaviors of nanocomposites prepared with oleic acid-modified clays are similar to those of extruded PET. Significant improvements in the mechanical and barrier properties of oriented PET/clay nanocomposites were also achieved. Well-dispersed modified clays acted as a passive barrier and improved the barrier properties of the films significantly. This improvement might be also a result of the reaction between the scavenger present in the gallery spacing of nanoparticles and oxygen.

\section{Conflicts of Interest}

The authors declare that they have no conflicts of interest.

\section{Acknowledgments}

The authors gratefully acknowledge PET research consortium for their kind support of this research.

\section{References}

[1] F. Awaja and D. Pavel, "Recycling of PET," European Polymer Journal, vol. 41, no. 7, pp. 1453-1477, 2005.

[2] T. Rieckmann and S. Völker, "Poly(ethylene terephthalate) polymerization-mechanism, catalysis, kinetics, mass transfer and reactor design," in Modern Polyesters: Chemistry and Technology of Polyesters and Copolyesters, pp. 29-115, John Wiley \& Sons, Ltd, 2004.

[3] M. Frounchi and A. Dourbash, "Oxygen barrier properties of poly(ethylene terephthalate) nanocomposite films," Macromolecular Materials and Engineering, vol. 294, no. 1, pp. 68-74, 2009.

[4] S. Hayrapetyan, A. Kelarakis, L. Estevez et al., "Non-toxic poly(ethylene terephthalate)/clay nanocomposites with enhanced barrier properties," Polymer, vol. 53, no. 2, pp. 422-426, 2012.

[5] M. R. Galdi, V. Nicolais, L. Di Maio, and L. Incarnato, "Production of active PET films: evaluation of scavenging activity," 
Packaging Technology and Science, vol. 21, no. 5, pp. 257-268, 2008.

[6] K. Mahajan, E. A. Lofgren, and S. A. Jabarin, "Development of active barrier systems for poly(ethylene terephthalate)," Journal of Applied Polymer Science, vol. 129, no. 4, pp. 2196-2207, 2013.

[7] H. Ghasemi, P. J. Carreau, M. R. Kamal, and J. Uribe-Calderon, "Preparation and characterization of PET/clay nanocomposites by melt compounding," Polymer Engineering and Science, vol. 51, no. 6, pp. 1178-1187, 2011.

[8] J.-H. Chang, S. J. Kim, Y. L. Joo, and S. Im, "Poly(ethylene terephthalate) nanocomposites by in situ interlayer polymerization: the thermo-mechanical properties and morphology of the hybrid fibers," Polymer, vol. 45, no. 3, pp. 919-926, 2004.

[9] A. Okada and A. Usuki, "Twenty years of polymer-clay nanocomposites," Macromolecular Materials and Engineering, vol. 291, no. 12, pp. 1449-1476, 2006.

[10] D. R. Paul and L. M. Robeson, "Polymer nanotechnology: nanocomposites," Polymer, vol. 49, no. 15, pp. 3187-3204, 2008.

[11] R. K. Bharadwaj, A. R. Mehrabi, C. Hamilton et al., "Structureproperty relationships in cross-linked polyester-clay nanocomposites," Polymer, vol. 43, no. 13, pp. 3699-3705, 2002.

[12] P. J. Yoon, T. D. Fornes, and D. R. Paul, "Thermal expansion behavior of nylon 6 nanocomposites," Polymer, vol. 43, no. 25, pp. 6727-6741, 2002.

[13] Y. Kojima, K. Fukumori, A. Usuki, A. Okada, and T. Kurauchi, "Gas permeabilities in rubber-clay hybrid," Journal of Materials Science Letters, vol. 12, no. 12, pp. 889-890, 1993.

[14] C. H. Davis, L. J. Mathias, J. W. Gilman et al., "Effects of melt-processing conditions on the quality of poly(ethylene terephthalate) montmorillonite clay nanocomposites," Journal of Polymer Science, Part B: Polymer Physics, vol. 40, no. 23, pp. 2661-2666, 2002.

[15] M. Wang, T. C. Chung, J. W. Gilman, and E. Manias, "Melt-processable syndiotactic polystyrene/montmorillonite nanocomposites," Journal of Polymer Science, Part B: Polymer Physics, vol. 41, no. 24, pp. 3173-3187, 2003.

[16] T. U. Patro, D. V. Khakhar, and A. Misra, "Phosphonium-based layered silicate-poly(ethylene terephthalate) nanocomposites: Stability, thermal and mechanical properties," Journal of Applied Polymer Science, vol. 113, no. 3, pp. 1720-1732, 2009.

[17] C. F. Ou, M. T. Ho, and J. R. Lin, "Synthesis and characterization of poly(ethylene terephthalate) nanocomposites with organoclay," Journal of Applied Polymer Science, vol. 91, no. 1, pp. 140-145, 2004.

[18] K. H. Kim, K. H. Kim, J. Huh, and W. H. Jo, "Synthesis of thermally stable organosilicate for exfoliated poly(ethylene terephthalate) nanocomposite with superior tensile properties," Macromolecular Research, vol. 15, no. 2, pp. 178-184, 2007.

[19] M. C. Costache, M. J. Heidecker, E. Manias, and C. A. Wilkie, "Preparation and characterization of poly(ethylene terephthalate)/clay nanocomposites by melt blending using thermally stable surfactants," Polymers for Advanced Technologies, vol. 17, no. 9-10, pp. 764-771, 2006.

[20] T. L. P. G. M. Booth and C. M. Lee, An Approach on Food Preservation, InTech, 2012.

[21] K. Majdzadeh-Ardakani and S. A. Jabarin, "Improving ther barrier and mechanical properties of PET/Clay nanocomposites, ANTEC SPE," Indianapolis, pp. 425-428, 2016.

[22] S. Rooj, A. Das, K. W. Stöckelhuber et al., "Pre-intercalation of long chain fatty acid in the interlayer space of layered silicates and preparation of montmorillonite/natural rubber nanocomposites," Applied Clay Science, vol. 67-68, pp. 50-56, 2012.
[23] S. A. Jabarin, "Strain-induced crystallization of poly(ethylene terephthalate)," Polymer Engineering \& Science, vol. 32, no. 18, pp. 1341-1349, 1992.

[24] S. Zekriardehani, S. A. Jabarin, D. R. Gidley, and M. R. Coleman, "Effect of chain dynamics, crystallinity, and free volume on the barrier properties of poly(ethylene terephthalate) biaxially oriented films," Macromolecules, vol. 50, no. 7, pp. 2845-2855, 2017.

[25] S. Al-AbdulRazzak and S. A. Jabarin, "Processing characteristics of poly(ethylene terephthalate): hydrolytic and thermal degradation," Polymer International, vol. 51, no. 2, pp. 164-173, 2002.

[26] N. H. Kim, S. V. Malhotra, and M. Xanthos, "Modification of cationic nanoclays with ionic liquids," Microporous and Mesoporous Materials, vol. 96, no. 1-3, pp. 29-35, 2006.

[27] G. M. Do Nascimento, V. R. L. Constantino, R. Landers, and M. L. A. Temperini, "Aniline polymerization into montmorillonite clay: a spectroscopic investigation of the intercalated conducting polymer," Macromolecules, vol. 37, no. 25, pp. 9373-9385, 2004.

[28] S. Rooj, A. Das, K. W. Stöckelhuber, U. Reuter, and G. Heinrich, "Highly exfoliated natural rubber/Clay composites by "propping-open procedure": the influence of fatty-acid chain length on exfoliation," Macromolecular Materials and Engineering, vol. 297, no. 4, pp. 369-383, 2012.

[29] E.-A. McGonigle, J. H. Daly, S. Gallagher et al., "Physical ageing in poly(ethylene terephthalate)-its influence on cold crystallisation," Polymer, vol. 40, no. 17, pp. 4977-4982, 1999.

[30] X. Yuan, C. Li, G. Guan, Y. Xiao, and D. Zhang, “Thermal stability of surfactants with amino and imido groups in poly(ethylene terephthalate)/clay composites," Journal of Applied Polymer Science, vol. 109, no. 6, pp. 4112-4120, 2008.

[31] C. I. W. Calcagno, C. M. Mariani, S. R. Teixeira, and R. S. Mauler, "The effect of organic modifier of the clay on morphology and crystallization properties of PET nanocomposites," Polymer, vol. 48, no. 4, pp. 966-974, 2007.

[32] Y. Wang and S. A. Jabarin, "Novel preparation method for enhancing nanoparticle dispersion and barrier properties of poly(ethylene terephthalate) and poly(m-xylylene adipamide)," Journal of Applied Polymer Science, vol. 129, no. 3, pp. 1455-1465, 2013. 

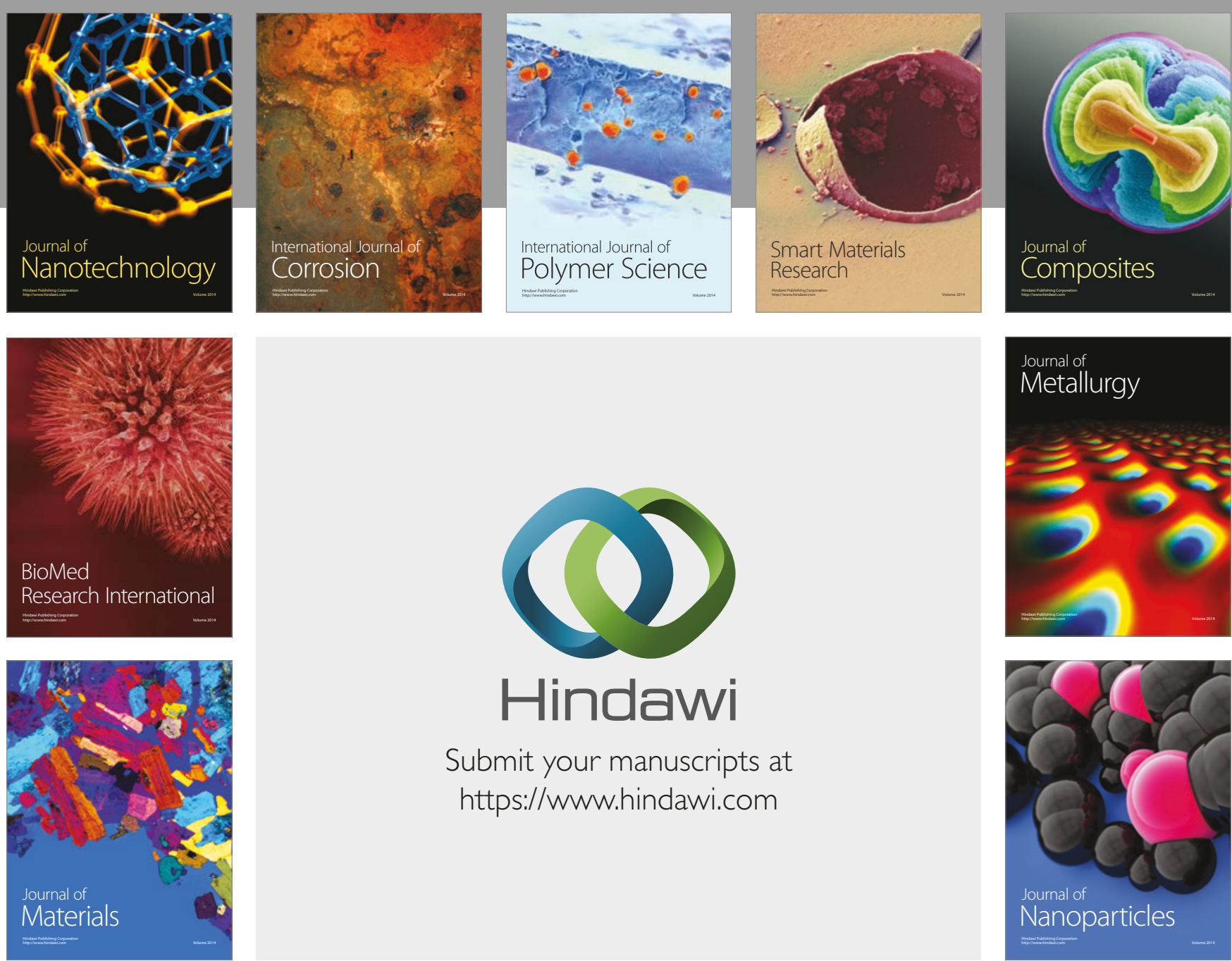

\section{Hindawi}

Submit your manuscripts at

https://www.hindawi.com
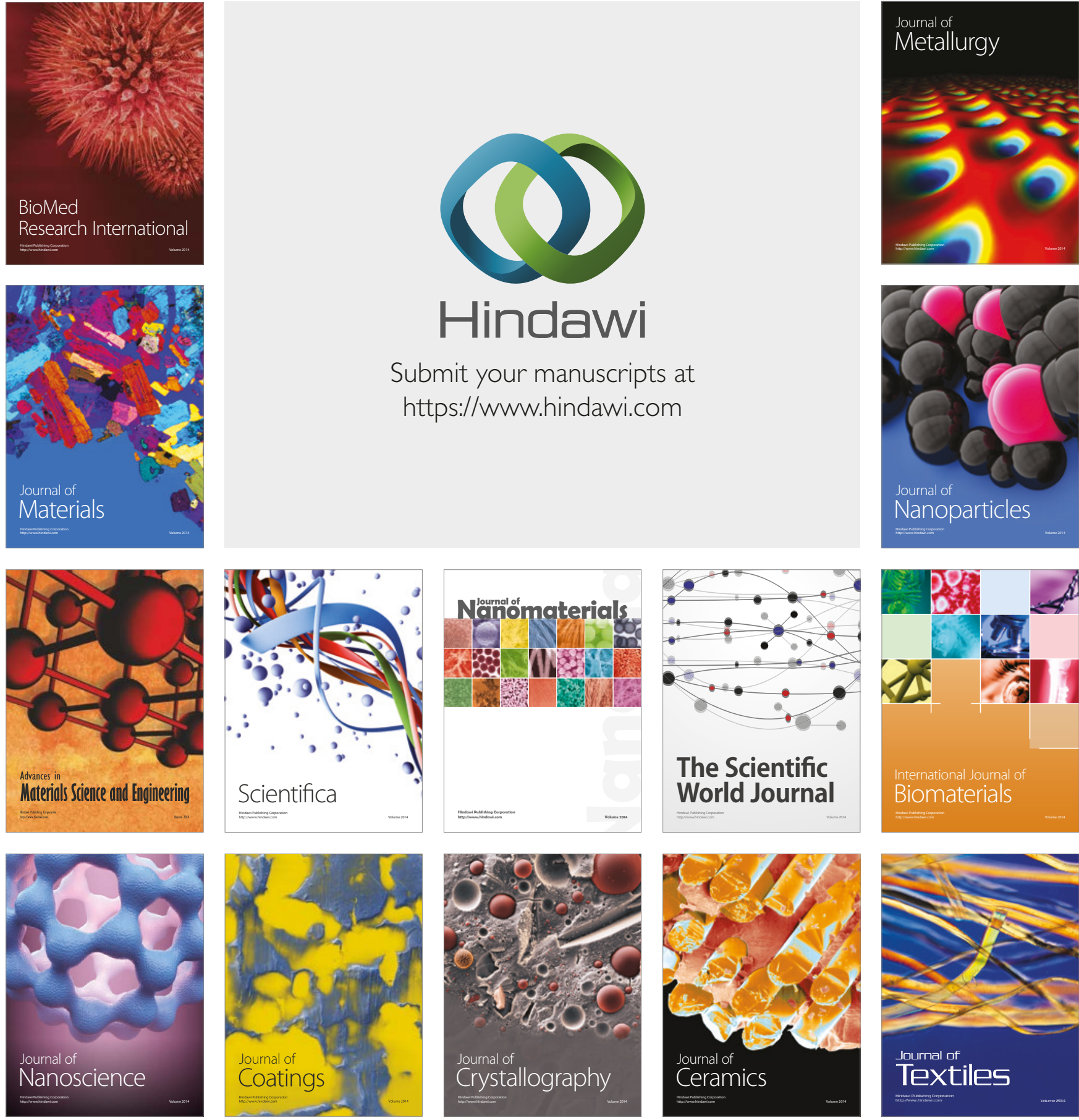

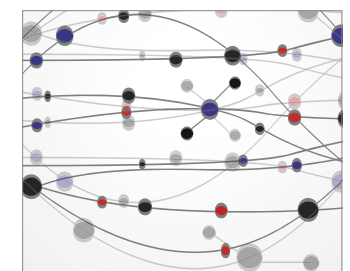

The Scientific World Journal
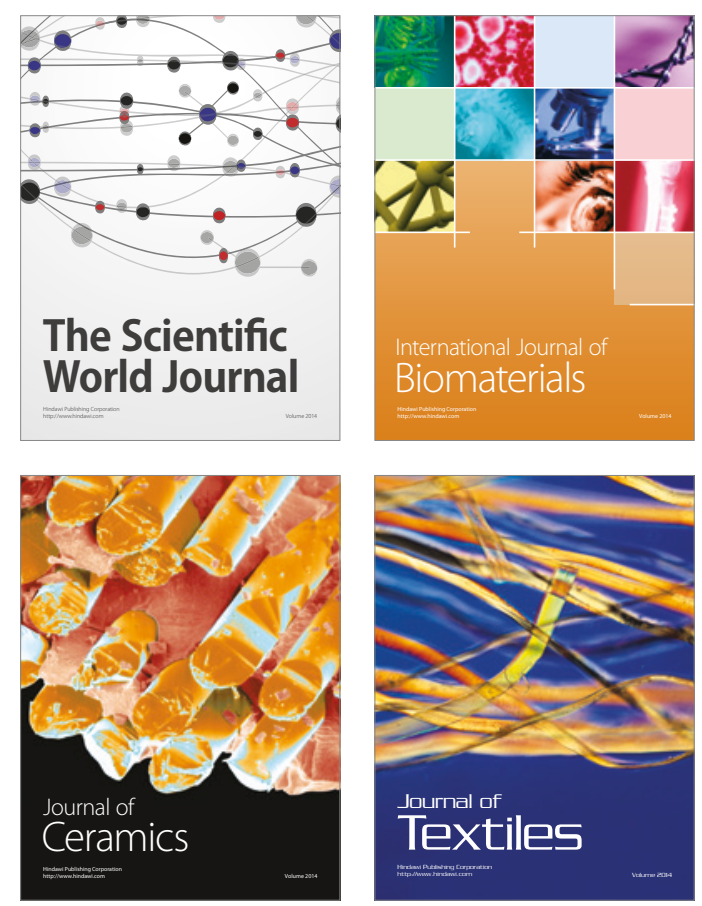